Bull. Austral. Math. Soc.

$37 \mathrm{C} 50,37 \mathrm{D} 20$

VOL. 69 (2004) [353-359]

\title{
INVERSE SHADOWING OF CIRCLE MAPS
}

\author{
Jong-Jin PARK AND KeonheE LeE
}

We show that the concepts of shadowing and $\left(\mathcal{T}_{c}, \mathcal{T}_{h}-\right)$ inverse shadowing of circle homeomorphisms are equivalent.

\section{INTRODUCTION}

When simulating the behaviour of a dynamical system we often encounter the following problems.

1. Does the orbit displayed on the computer screen really correspond to some true orbit?

2. Can every true orbit be recovered, at least with a given accuracy?

The first problem is in fact a question about the shadowing property of the system while the second one corresponds to the property known as inverse shadowing. Shadowing, or the pseudo orbit tracing property, was first established for systems generated by hyperbolic diffeomorphisms and later for those generated by hyperbolic homeomorphisms. It says that any $\delta$-pseudo orbit can be uniformly approximated by a true orbit with a given accuracy if $\delta>0$ is sufficiently small.

Recently inverse shadowing was established by Corless and Pilyugin [1] and also as a part of the concept of bishadowing by Diamond, Kloeden, Kozyakin and Pokrovskii [2]. Kloeden and Ombach [5] redefined this property using the concept of a $\delta$-method. Generally speaking, a dynamical system is inverse shadowing with respect to a class of methods if any true orbit can be uniformly approximated with given accuracy by a $\delta$ pseudo orbit generated by a method from the chosen class if $\delta>0$ is sufficiently small. An appropriate choice of the class of admissible pseudo orbits is crucial here (see $[1,3,5,7]$ ).

There are some results about how shadowing and inverse shadowing are related each other (for more details, see $[3,5,6,7]$ ).

It is well known that every shift homeomorphism is both expansive and shadowing, but it is not $\mathcal{T}_{c}$-inverse shadowing (see [5, Example 3]).

Received 28th November, 2002

This work was supported by grant No. F01-2001-000-20002-0, R01-2000-000-00005-0(2003) from the Korea Science and Engineering Foundation.

Copyright Clearance Centre, Inc. Serial-fee code: 0004-9727/04 \$A2.00+0.00. 
On the other hand, we see that any pseudo Anosov homeomorphism on compact surfaces is both expansive and $\mathcal{T}_{h}$-inverse shadowing (persistent). However, in general, it is not shadowing. In fact, every pseudo Anosov homeomorphism on compact surfaces need not be topologically stable (see $[4,8]$ ).

In this paper we show that the concepts of shadowing and $\left(\mathcal{T}_{c}, \mathcal{T}_{h}-\right)$ inverse shadowing of circle homeomorphisms are equivalent.

\section{INVERSE SHADOWING}

Let $X$ be a compact metric space with a metric $d$, and let $Z(X)$ denote the space of homeomorphisms on $X$ with the $C_{0}$-metric $d_{0}$. A homeomorphism $f \in Z(X)$ will be identified with the dynamical system it generates by iteration.

A $\delta$-pseudo orbit of $f \in Z(X)$ is a sequence of points $\xi=\left\{x_{k} \in X: k \in \mathbb{Z}\right\}$ such that $d\left(f\left(x_{k}\right), x_{k+1}\right)<\delta$ for all $k \in \mathbb{Z}$. A $\delta$-pseudo orbit $\xi=\left\{x_{k}\right\}$ is said to be $\varepsilon$-shadowed by a point $x \in X$ (or an orbit $\left\{f^{k}(x): k \in \mathbb{Z}\right\}$ ) if $d\left(f^{k}(x), x_{k}\right)<\varepsilon$ for all $k \in \mathbb{Z}$. Say that $f \in Z(X)$ is shadowing (or pseudo orbit tracing property) if given $\varepsilon>0$ there exists $\delta>0$ such that any $\delta$-pseudo orbit of $f$ is $\varepsilon$-shadowed by a point (or an orbit) in $X$.

Let $X^{\mathbf{Z}}$ be the compact metric space of all two sided sequences $\xi=\left\{x_{k}: k \in \mathbb{Z}\right\}$ in $X$, endowed with the product topology. For a constant $\delta>0$ and $f \in Z(X)$, let $\Phi_{f}(\delta)$ denote the set of all $\delta$-pseudo orbits of $f$.

A mapping $\varphi: X \rightarrow \Phi_{f}(\delta) \subset X^{\mathbf{Z}}$ satisfying $\varphi_{0}(x)=x, x \in X$, is said to be a $\delta$-method for $f$. For convenience, write $\varphi(x)$ for $\left\{\varphi_{k}(x)\right\}_{k \in \mathbf{Z}}$. Say that $\varphi$ is a continuous $\delta$-method for $f$ if $\varphi$ is continuous. The set of all $\delta$-methods [respectively continuous $\delta$-methods] for $f$ will be denoted by $\mathcal{T}_{0}(f, \delta)$ (respectively $\mathcal{T}_{c}(f, \delta)$ ). Every $g \in Z(X)$ with $d_{0}(f, g)<\delta$ induces a continuous $\delta$-method $\varphi_{g}: X \rightarrow X^{\mathbf{Z}}$ for $f$ by defining $\varphi_{g}(x)$ $=\left\{g^{k}(x): k \in \mathbb{Z}\right\}$. Let $\mathcal{T}_{h}(f, \delta)$ denote the set of all continuous $\delta$-methods $\varphi_{g}$ for $f$ which are induced by $g \in Z(X)$ with $d_{0}(f, g)<\delta$. Then we have

$$
\mathcal{T}_{h}(f, \delta) \subset \mathcal{T}_{c}(f, \delta) \subset \mathcal{T}_{0}(f, \delta)
$$

Note that a method in $\mathcal{T}_{c}(f, \delta)$ need not be generated by a single mapping.

Definition: $f \in Z(X)$ is said to be $\mathcal{T}_{\alpha}$-inverse shadowing, $\alpha=0, c, h$, if for any $\varepsilon>0$ there exists $\delta>0$ such that for any f-orbit $\xi=\left\{x_{k}\right\}$ and any $\delta$-method $\varphi \in \mathcal{T}_{\alpha}(f, \delta)$ there is $y \in X$ such that

$$
d\left(x_{k}, \varphi_{k}(y)\right)<\varepsilon
$$

for all $k \in \mathbb{Z}$.

Clearly we have the following relations among three notions of inverse shadowing.

$\mathcal{T}_{0}$ - inverse shadowing $\Rightarrow \mathcal{T}_{c}$ - inverse shadowing $\Rightarrow \mathcal{T}_{h}$ - inverse shadowing. 
In [8], Lewowicz introduced a notion of persistency for a homeomorphism $f \in Z(X)$; and he showed that any pseudo Anosov homeomorphism on compact surfaces is persistent. Note that $\mathcal{T}_{h}$-inverse shadowing here is equivalent to persistency in $[8,12]$.

We can easily see that two concepts of shadowing and $\mathcal{T}_{0}$-inverse shadowing of circle homeomorphisms are not equivalent. In fact, let $f$ be a homeomorphism on the unit circle $S^{1}$, with coordinate $x \in[0,1)$, given by $f(x)=x+(1 / 2 \pi) \sin (2 \pi x)$. Then it is shadowing, but it is not $\mathcal{T}_{0}$-inverse shadowing.

THEOREM. For any $f \in Z\left(S^{1}\right)$, the following statements are equivalent:

(A) $f$ is shadowing (pseudo orbit tracing property);

(B) $f$ is $\mathcal{T}_{c}$-inverse shadowing;

(C) $f$ is $\mathcal{T}_{h}$-inverse shadowing (persistent).

To prove our theorem, some further notations and known results are required.

Consider the circle $S^{1}$ with coordinate $x \in[0,1)$, and we denote by $d$ the metric on $S^{1}$ induced by the usual distance on the real line. It is easy to show that $f \in Z\left(S^{1}\right)$ is $\mathcal{T}_{\alpha}$-inverse shadowing [respectively shadowing] if and only if $f^{k}$ is $\mathcal{T}_{\alpha}$-inverse shadowing [respectively shadowing] for some $k \in \mathbb{Z}-\{0\}$, where $\alpha=c, h$. Due to these facts, when studying the theory of shadowing and inverse shadowing, attention can be restricted to those homeomorphisms on $S^{1}$ which preserve orientation.

Let $\pi: \mathbb{R} \rightarrow S^{1}$ be the covering projection defined by the relations

$$
\pi(x) \in[0,1), \pi(x) \equiv x(\bmod 1)
$$

with respect to the considered coordinates on $S^{1}$. Let $f \in Z\left(S^{1}\right)$ and $F: \mathbb{R} \rightarrow \mathbb{R}$ the lift of $f$ such that $F(0) \in[0,1)$. It is well known that for any $x \in \mathbb{R}$ the limit

$$
\mu(f)=\lim _{k \rightarrow \infty}=\frac{F^{k}(x)}{k}(\bmod 1)
$$

exists and does not depend on $x$. This quantity is called the rotation number of $f$ and measures the average amount that a point in $S^{1}$ is rotated by $f$. The main property of the rotation number is that $f$ has a periodic point if and only if $\mu(f)$ is rational (see $[9,10])$.

For any two points $a, b \in S^{1}$, we denote by $(a, b)$ the open arc of $S^{1}$ corresponding to the set $(a, b) \subset[0,1)$ if $a<b$, and to the set $(a, 1) \cup[0, b) \subset[0,1)$ if $b<a$. Similar notation is applied for closed arcs.

For $f \in Z\left(S^{1}\right)$ we denote by $\operatorname{Fix}(f)$ the set of fixed points of $f$. Recently Plamenevskaya [11] gave necessary and sufficient conditions for which $f \in Z\left(S^{1}\right)$ is shadowing.

LEMMA. ([11].) $f \in Z\left(S^{1}\right)$ is shadowing if and only if $f$ satisfy the following two conditions: 
(1) Fix $(f)$ is nowbere dense and contains at least two points;

(2) for any $a, b \in \operatorname{Fix}(f)$, either $\operatorname{Fix}(f) \cap(a, b)=\emptyset$ or the function $F(t)-t$ changes sign on $(a, b)$, where $F$ is the lift of $f$ with $F(0) \in[0,1)$.

Now we prove our theorem using the above lemma. Obviously, the implication $(B) \Rightarrow(C)$ holds. We prove here that $(A) \Rightarrow(B)$ and that $(C) \Rightarrow(A)$.

Proof of $(\mathrm{A}) \Rightarrow(\mathrm{B})$ : Suppose $f \in Z\left(S^{1}\right)$ is shadowing, and let $\varepsilon>0$ be arbitrary. By applying the above lemma, we can choose fixed points $a_{1}, b_{1}, \ldots, a_{2 N}, b_{2 N}$ of $f$ such that

(1) $0 \leqslant a_{1}<b_{1} \leqslant a_{2}<b_{2} \leqslant \cdots \leqslant a_{2 N}<b_{2 N} \leqslant 1$;

(2) the length of any segment $\left[b_{1}, a_{2}\right], \ldots,\left[b_{2 N}, a_{1}\right]$ is less than $\varepsilon$;

(3) $F(t)-t \neq 0$ on $\left(a_{n}, b_{n}\right), 1 \leqslant n \leqslant 2 N$;

(4) if $F(t)-t>0$ on $\left(a_{n}, b_{n}\right)$ then $F(t)-t<0$ on $\left(a_{n+1}, b_{n+1}\right)$ and vice versa, where $1 \leqslant n \leqslant 2 N, a_{2 N+1}=a_{1}$ and $b_{2 N+1}=b_{1}$.

For convenience we assume that if $n$ is odd then $F(t)-t>0$ on $\left(a_{n}, b_{n}\right)$, and that if $n$ is even then $F(t)-t<0$ on $\left(a_{n}, b_{n}\right)$. For each $1 \leqslant n \leqslant 2 N$, we take points $a_{n}^{\prime}, b_{n}^{\prime} \in[0,1)$ such that

(1) $a_{n}<a_{n}^{\prime}<b_{n}^{\prime}<b_{n}$ and $d\left(\left[b_{n}^{\prime}, a_{n+1}^{\prime}\right]\right)<\varepsilon$;

(2) $f\left(\left[b_{n}^{\prime}, a_{n+1}^{\prime}\right]\right) \cap\left[b_{m}^{\prime}, a_{m+1}^{\prime}\right]=\emptyset$ if $m \neq n$, where $a_{2 N+1}^{\prime}=a_{1}^{\prime}$.

Let $I_{n}=\left[a_{n}^{\prime}, b_{n}^{\prime}\right]$ and $I_{n}^{\prime}=\left[b_{n}^{\prime}, a_{n+1}^{\prime}\right]$ for each $1 \leqslant n \leqslant 2 N$. Choose $\alpha>0$ satisfying

$$
d(f(x), x)>\alpha \text { and } d\left(f^{-1}(x), x\right)>\alpha
$$

for $x \in \bigcup_{n=1}^{2 N} I_{n}$. Let $\beta>0$ be such that

(1) $\beta<\min \{\varepsilon,(\alpha / 2)\}$ and $d\left(f\left(I_{n}^{\prime}\right), I_{m}^{\prime}\right)>\beta$ if $m \neq n$;

(2) $\mathcal{N}_{\beta}\left(f\left(I_{n}^{\prime}\right)\right) \subset \operatorname{int}\left(I_{n}^{\prime}\right)$ if $n$ is odd, and $\mathcal{N}_{\beta}\left(f^{-1}\left(I_{n}^{\prime}\right)\right) \subset \operatorname{int}\left(I_{n}^{\prime}\right)$ if $n$ is even.

Let $L>0$ be an integer with $2 / \beta \leqslant L<(2 / \beta)+1$. For each $i=1, \ldots, L$, we take $r_{i}>0$ such that

(1) $0<r_{L}<\cdots<r_{1}<r_{0}=\beta$;

(2) if $d(x, y)<r_{i}$ then $\max \left\{d(f(x), f(y)), d\left(f^{-1}(x), f^{-1}(y)\right)\right\}<r_{i-1}$.

Select $r>0$ satisfying $L r<r_{L}$. Let $\delta>0$ be such that if $d(x, y)<\delta$ then $d\left(f^{k}(x), f^{k}(y)\right)<r$ for all $-L \leqslant k \leqslant L$.

Let $\varphi$ be any continuous $\delta$-method for $f$, and let $\varphi_{k}$ be the $k$-th coordinate function of $\varphi$ for $k \in \mathbb{Z}$. Then we can easily check that

$$
d\left(f^{k}(p), \varphi_{k}(p)\right)<\varepsilon
$$

for all $p \in S^{1}$ and all $|k| \leqslant L$. Let $x \in S^{1}$. To show that $f$ is $\mathcal{T}_{c}$-inverse shadowing, we have to find $k_{0} \in \mathbb{Z}$ and $y_{0} \in S^{1}$ satisfying

$$
d\left(f^{k}\left(f^{k_{0}}(x)\right), \varphi_{k}\left(y_{0}\right)\right)<\varepsilon
$$


for all $k \in \mathbb{Z}$.

First we suppose that $\left\{f^{k}(x): k \in \mathbb{Z}\right\} \cap I_{n} \neq \emptyset$ for some $1 \leqslant n \leqslant 2 N$. Assume that $f^{k_{0}}(x) \in I_{n}$ for some $k_{0} \in \mathbb{Z}$ and that $n$ is odd. In this case, we put $y_{0}=f^{k_{0}}(x)$. By our constructions, we have

$$
f^{k}\left(f^{k_{0}}(x)\right), \varphi_{k}\left(y_{0}\right) \in I_{n}^{\prime} \text { and } f^{-k}\left(f^{k_{0}}(x)\right), \varphi_{-k}\left(y_{0}\right) \in I_{n-1}^{\prime}
$$

if $k \geqslant L$. Since (a) holds for $|k| \leqslant L$, (b) implies that $(*)$ holds for all $k \in \mathbb{Z}$.

Next we suppose that $\left\{f^{k}(x): k \in \mathbb{Z}\right\} \subset I_{n}^{\prime}$ for some $1 \leqslant n \leqslant 2 N$. Assume that $n$ is odd and that $0<b_{n}^{\prime}<b_{n} \leqslant a_{n+1}<a_{n+1}^{\prime}<1$. Let $y \in I_{n}^{\prime} \subset[0,1)$. Then we have $\varphi_{k}(y) \in I_{n}^{\prime}$ for all $k \geqslant 0$. Let

$$
\begin{aligned}
& \mathcal{U}=\left\{y \in I_{n}^{\prime}: \varphi_{k}(y)>a_{n+1}^{\prime} \text { for some } k<0\right\}, \\
& \mathcal{V}=\left\{y \in I_{n}^{\prime}: \varphi_{k}(y)<b_{n}^{\prime} \text { for some } k<0\right\}
\end{aligned}
$$

Then $\mathcal{U}$ and $\mathcal{V}$ are nonempty open sets, and $\mathcal{U} \cap \mathcal{V}=\emptyset$. In fact, if $\varphi_{k}(y)>a_{n+1}^{\prime}$ for some $k<0$ then we can see that $\varphi_{s}(y)>a_{n+1}^{\prime}$ for all $s \leqslant k$; and if $\varphi_{k}(y)<b_{n}^{\prime}$ for some $k<0$ then we have that $\varphi_{s}(y)<b_{n}^{\prime}$ for all $s \leqslant k$. This means that $\mathcal{U} \cap \mathcal{V}=\emptyset$. For any $y \in \mathcal{U} \subset I_{n}^{\prime}$, we have $\varphi_{k}(y)>a_{n+1}^{\prime}$ for some $k<0$. Since $\varphi_{k}$ is continuous, there exists a neighbourhood $\mathcal{W}$ of $y$ in $I_{n}^{\prime}$ such that $\varphi_{k}(z)>a_{n+1}^{\prime}$ for all $z \in \mathcal{W}$. This implies that $\mathcal{W} \subset \mathcal{U}$. Since $\mathcal{U} \cup \mathcal{V} \neq I_{n}^{\prime}$, we can find a point $y_{0} \in I_{n}^{\prime} /(\mathcal{U} \cup \mathcal{V})$. For the point $y_{0}$, we have

$$
d\left(f^{k}(x), \varphi_{k}\left(y_{0}\right)\right)<\varepsilon
$$

for all $k \in \mathbb{Z}$; and so completes the proof of $(A) \Rightarrow(B)$.

Proof of $(\mathrm{C}) \Rightarrow(\mathrm{A})$ : Suppose $f \in Z\left(S^{1}\right)$ is $\mathcal{T}_{h}$-inverse shadowing.

First we show that $f$ has periodic points. To obtain a contradiction, we assume that $f$ does not have periodic points. Then $\operatorname{Fix}(f)=\emptyset$, and we can choose $\varepsilon>0$ such that $d(f(x), x)>3 \varepsilon$ for $x \in S^{1}$. Let $0<\alpha<\varepsilon$ be a number given for this $\varepsilon>0$ by definition of $\mathcal{T}_{h}$-inverse shadowing. For any $x \in S^{1}$, we can choose distinct two integers $i, j \in \mathbb{Z}$ satisfying

$$
d\left(f^{i}(x), f^{j}(x)\right)<\frac{\alpha}{2}
$$

Assume that $i<j$, and let $x_{*}=f^{i}(x)$ and $n=j-i$. Then we have $d\left(x_{*}, f^{n}\left(x_{*}\right)\right)<\alpha / 2$. We may assume that $d\left(x_{*}, f^{k}\left(x_{*}\right)\right)>\alpha / 2$ for $0<k<n$. Choose $\delta>0, \alpha / 2<\delta<\alpha$, satisfying

$$
\left\{f\left(x_{*}\right), \ldots, f^{n-1}\left(x_{*}\right)\right\} \cap\left(x_{*}-\delta, x_{*}+\delta\right)=\emptyset .
$$

Let $h: S^{1} \rightarrow S^{1}$ be a homeomorphism such that

$$
h(x)= \begin{cases}x & \text { if } x \notin\left(x_{*}-\delta, x_{*}+\delta\right), \\ x_{*} & \text { if } x=f^{n}\left(x_{*}\right)\end{cases}
$$


Put $g=h \circ f$. Then we have

$$
g^{n}\left(x_{*}\right)=x_{*} \text { and } d_{0}(f, g)<\alpha .
$$

By assumption, there exists $y_{*} \in S^{1}$ such that

$$
d\left(f^{k}\left(x_{*}\right), g^{k}\left(y_{*}\right)\right)<\varepsilon
$$

for all $k \in \mathbb{Z}$. Choose $x_{0}, y_{0} \in[0,1) \subset \mathbb{R}$ satisfying $\pi\left(x_{0}\right)=x_{*}$ and $\pi\left(y_{0}\right)=y_{*}$. Let $F$ and $G$ be the lifts of $f$ and $g$, respectively, satisfying $F(0), G(0) \in[0,1)$. For each $k \in \mathbb{Z}$, choose $y_{k} \in \mathbb{R}$ such that

$$
\pi\left(y_{k}\right)=g^{k}\left(y_{*}\right) \text { and } y_{k}<y_{k+1}<y_{k}+1
$$

Since $\operatorname{Fix}(f)=\emptyset, F(t)-t \neq \emptyset$ for $t \in[0,1)$ and $F(0) \in[0,1)$, we have that $0<F(t)-t$ $<1$ for all $t \in \mathbb{R}$. Using the above facts, we can show that

$$
\left|F^{k}\left(x_{0}\right)-y_{k}\right|<\varepsilon
$$

for all $k \in \mathbb{Z}$. Then we have

$$
\mu(f)=\lim _{k \rightarrow \infty} \frac{F^{k}\left(x_{0}\right)}{k}=\lim _{k \rightarrow>\infty} \frac{y_{k} / k}{=} \lim _{k \rightarrow \infty} \frac{\left(G^{k}\left(y_{0}\right)\right.}{k}=\mu(g),
$$

and so $\mu(f)$ is rational. Moreover $\mathcal{T}_{h}$-inverse shadowing of $f$ implies that $f$ has at least two periodic points.

Next we show that if $(a, b) \cap \operatorname{Fix}(f) \neq \emptyset$ for $a, b \in \operatorname{Fix}(f)$ with $a<b$, then the function $F(t)-t$ changes sign on $(a, b)$. To see this, suppose $F(t)-t \geqslant 0$ for all $t \in(a, b)$, and let $c \in(a, b) \cap \operatorname{Fix}(f)$. Choose $\varepsilon>0$ such that $\varepsilon<1 / 2 \min \{d(a, c), d(c, b)\}$. For any $\delta>0$, there exists $g_{\delta} \in Z(S)$ such that

$$
d_{0}\left(f, g_{\delta}\right)<\delta \text { and } G_{\delta}(t)-t>0
$$

for $t \in(a, b)$, where $G_{\delta}$ is the lift of $g_{\delta}$ with $G_{\delta}(0) \in[0,1)$. However, then no $f$-orbit in $(a, b)$ can $\varepsilon$-shadows the $g_{\delta}$-orbit through $c$, and this contradiction shows that the function $F(t)-t$ changes sign on $(a, b)$. Application of Lemma completes the proof of $(C) \Rightarrow(A)$.

\section{REFERENCES}

[1] R. Corless and S. Pilyugin, 'Approximate and real trajectories for generic dynamical systems', J. Math. Anal. Appl. 189 (1995), 409-423.

[2]. P. Diamond, P. Kloeden, V.Kozyakin and A. Pokrovskii, 'Computer robustness of semi-hyperbolic mappings', Random Comput. Dynam. 3 (1995), 57-70.

[3] P. Diamond, Y. Han and K. Lee, 'Bishadowing and hyperbolicity', Internat. J. Bifur. Chaos Appl. Sci. Engrg. 12 (2002), 1779-1788. 
[4] M. Handel, 'Global shadowing of pseudo Anosov homeomorphisms', Ergodic Theory Dynamical Systems 5 (1985), 373-377.

[5] P. Kloeden and J. Ombach, 'Hyperbolic homeomorphisms and bishadowing', Ann. Polon. Math. 65 (1997), 171-177.

[6] P. Kloeden, J. Ombach and A. Pokrovskii, 'Continuous and inverse shadowing', Funct. Differ. Equ. 6 (1999), 137-153.

[7] K. Lee, 'Continuous inverse shadowing and hyperbolicity', Bull. Austral. Math. Soc. 67 (2003), 15-26.

[8] J. Lewowicz, 'Persistence in expensive systems', Ergodic Theory Dynamical Systems 3 (1983), 567-578.

[9] W. Melo and S. Strien, One-dimensional dynamics (Springer-Verlag, Berlin, 1993).

[10] S. Pilyugin, Shadowing of dynamical systms, Lecture Notes in Math. 1706 (Springer-Verlag, Berlin, Heidelberg, New York, 1999).

[11] O. Plamenevskaya, 'Pseudo-orbit tracing property and limit shadowing property on a circle', Vestnik St. Petersburg Univ. Math. 30 (1997), 27-30.

[12] K. Sakai, 'Diffeomorphisms with persistency', Proc. Amer. Math. Soc. 124 (1996), 2249-2254.

Department of Mathematics

Chonbuk National University

Chonju, Chonbuk

Korea

e-mail: jjpark46@math.chonbuk.ac.kr
Department of Mathematics Chungnam National University

Daejeon, 305-764

Korea

e-mail: khlee@math.chungnam.ac.kr 